\title{
Demonstrating the AMazING Panel
}

\author{
João Martins \\ Instituto Telecomunicações \\ Universidade de Aveiro \\ Aveiro, Portugal \\ jmartins@av.it.pt
}

\author{
João Paulo Barraca \\ Instituto Telecomunicações \\ Universidade de Aveiro \\ Aveiro, Portugal \\ jpbarraca@ua.pt \\ Rui L. Aguiar \\ Instituto Telecomunicações \\ Universidade de Aveiro \\ Aveiro, Portugal \\ ruilaa@ua.pt
}

\author{
Diogo Gomes \\ Instituto Telecomunicações \\ Universidade de Aveiro \\ Aveiro, Portugal \\ dgomes@ua.pt
}

\section{Categories and Subject Descriptors}

C. 2 [Computer Communication Networks]: Network Operations; D. 2 [Software Engineering]: Management; H.4 [Information Systems Applications]: Miscellaneous

\section{General Terms}

Experimentation, Management, Measurement

\section{Keywords}

network, testbed, experimentation, web, wireless, omf

The Internet, and networks in general, are in many ways part of our daily life. As their usage increases, their faults and limitations also become more apparent, motivating network researchers to evaluate and develop new solutions anticipating future scenarios and overcoming the challenges identified. These challenges vary across a wide range of networking areas, from traffic optimization in telecom operators, to scalability in wide area networks and broadband multimedia streaming, or even to the contextualization of communications in order to optimize usage. For researchers to create reliable network solutions, able to be included in our every day life, these solutions must be carefully designed, tested, and then put into a cycle of successive refinement.

Currently, there are some testbed management systems available. The core of most existent solutions can be reduced to a couple of shell scripts to operate the testbed resources, without the proper support for experiment automation. Running experiments is thus possible and flexible, but cumbersome, labor intensive, and error prone. The existent solutions lack proper graphical interfaces facilitating user interaction, while centralizing the whole process of configuring experiments, reserving resources, scheduling, and collecting its results. Furthermore, collaboration of multiple parties is vital in the current research environment. Experiment results must be shared with a closed number of peers in order to allow a better analysis and a more solid validation inside research terms.

The AMazING testbed [1] is composed by twenty-four nodes located in the rooftop of Instituto de Telecomunicações - Aveiro. A customized version of OMF is used to provide testbed management functions, automating otherwise tedious but required tasks

Copyright is held by the author/owner(s). WiNTECH'12, August 22, 2012, Istanbul, Turkey. ACM 978-1-4503-1527-2/12/08. such as network configuration, software deployment, and experiment execution. In addition we further developed a management overlay centered on the experimenters' workflow, allowing users to create and schedule experiments, as well as analyze the results produced. This management overlay constitutes an extension to OMF that exposes in a user friendly way its functionality for operation in a testbed.

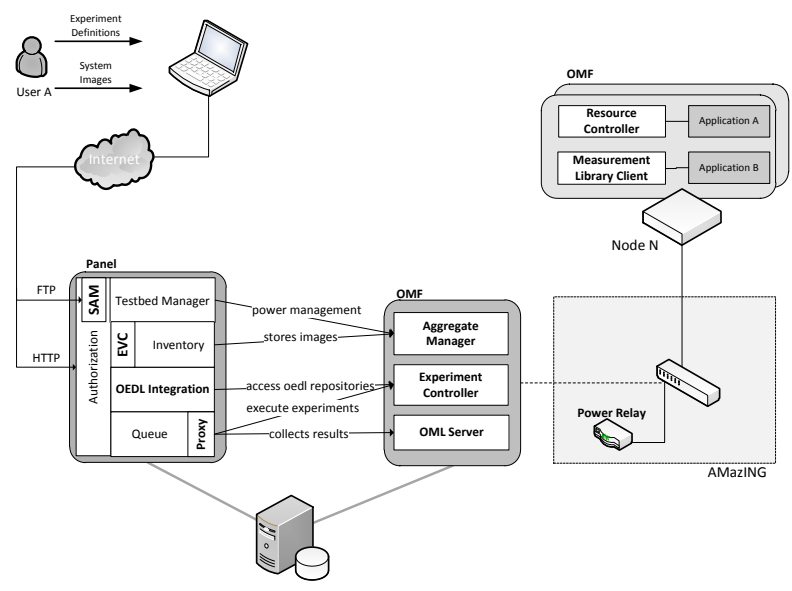

Figure 1: OMF architecture extensions

The Panel consists of an application that allows users to interact with the testbed through a graphical interface, while at the same time allowing administrators to manage users and some aspects of system operation. All OMF functionality is available through the interface created. Several modules were developed (see Figure 1) to create a scalable solution for the management system. A Testbed Manager controls the electrical power provided to each node. A FIFO Queue is used as the schedule mechanism for experiments execution. The AMazING usage model is "lease-time", meaning that at a given time the whole testbed is dedicated to a single experiment. A Proxy is responsible for the testbed operation, by preparing and executing experiments, on behalf of the users. Most importantly, it exposes OMF commands through a service oriented interface. The Integrated Experiments Environment (IEE) guides users in the development and execution of their experiments defini- 
tions. For new users of the platform, it simplifies the learning process when using an OMF-enabled testbed. The System Accounts Manager (SAM) is in charge of managing the FTP accounts. An Inventory represents the main data repository storing all the experiment definitions, system images and experiment related data are stored in this module. Finally, the Experiment Version Control (EVC) is responsible for supporting experiment versioning and the creation of branches.

We will highlight how the AMazING testbed management system can improve the process of creating, and executing an experiment, as well as gathering the results obtained. Provisioning of an experiment in the AMazING testbed system is divided in three steps: configuration of the experiment parameters and software deployed (see Figure 2); definition of the execution scenario, and finally configuration of the metrics to be observed and analyzed. After these steps, the experiment is queued for execution, probes are deployed, code is executed, and results are gathered. Finally, users can analyze the data obtained and, if required, branch the current experiment by creating variations of the conditions, which resulting data can then be compared. While the system developed makes use of OMF, it enhances OMF by facilitating the entire experimentation process.

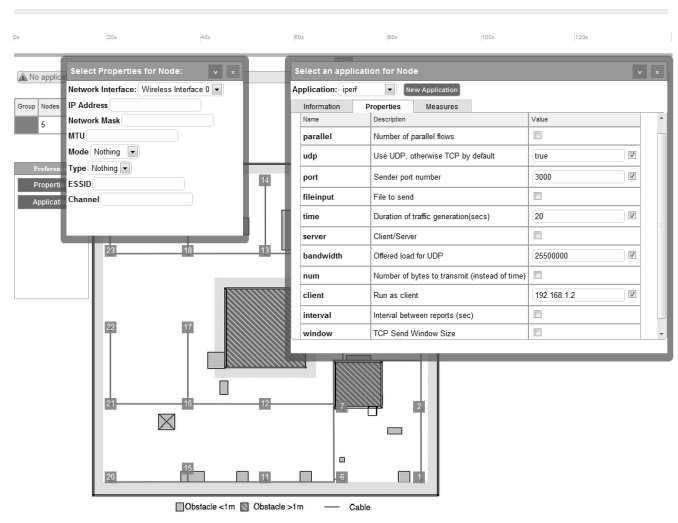

Figure 2: Configuring experiment parameters. Left sidebar represents the toolbox. Tables on top indicates nodes/applications configured

After the experiment is setup, it can be queued in the system, issuing either a single or multiple runs. Multiple runs are of particular importance for system analysis, and when evaluating applications over the highly dynamic wireless medium. Users can see the status of the process, and an email is sent once the experiment finishes.

Running an experiment can take several minutes or hours. When an experiment reaches its completion, the user navigates to the previously described environment for further analysis of the results obtained (see Figure 3). If an offline analysis is preferred, a database with all the results can be obtained and used in tools such as Matlab. The AMazING Panel allows a coarse analysis to be reduced to a few clicks.

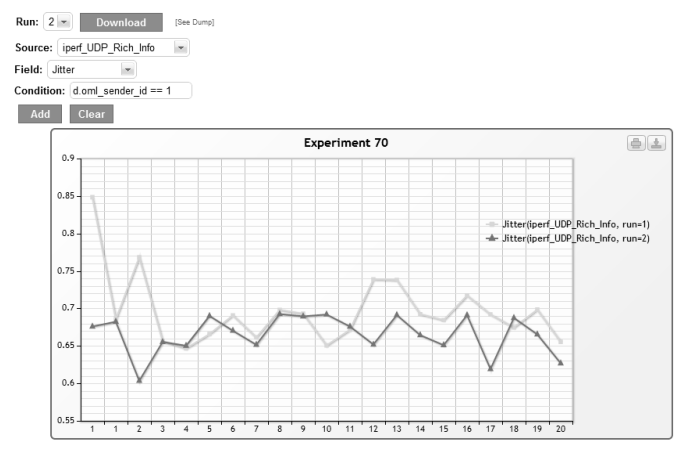

Figure 3: Graphics visualized include data sets from different runs or branches.

As our testbed tool usage grows, a broader range of OML integrated applications is expected to be available for users to use in their experiments (e.g. VLC for streaming, Mobile IP). Nevertheless, we already support most common tools such as Orbit Traffic Generator, IPerf and VLC.

In the near future we intend to stabilize and enhance the solution developed, adding support to a richer set of administrative tasks, as well as an integrated monitoring module for the purpose of monitoring operational metrics (CPU usage, Temperature, etc...) and node status (detect potential problems).

\section{REFERENCES}

[1] J. P. Barraca, D. Gomes, and R. L. Aguiar. AMazING Advanced Mobile wIreless playGrouNd. In International Conference on Testbeds and Research Infrastructures for the Development of Networks and Communities Proceeding, TRIDENTCOM '10, pages 219-230, 2010. 